\title{
On the structure of voting systems between two alternatives
}

\author{
Bonifacio Llamazares
}

Received: date / Accepted: date

\begin{abstract}
Voting systems between two alternatives have been widely studied in the literature of Social Choice. One of the results given by Fishburn (The Theory of Social Choice, Princeton University Press, 1973) allows us to characterize anonymous, neutral and monotonic voting systems by means of functions satisfying adequate conditions. From among all kinds of functions, the class of affine functions is highly interesting because from them it is possible to obtain the voting systems most used in practice. In this paper we analyze the structure of the set of these functions and we show that this set is convex and its extreme points are the functions that generate the following voting systems: simple majority, absolute majority, unanimous majority and Pareto majority. Moreover, we suggest a simple method for choosing a voting system when two alternatives are under consideration.
\end{abstract}

Keywords Voting systems · Simple majority · Absolute majority · Unanimous majority $\cdot$ Pareto majority

\section{JEL Classification D71}

\section{Introduction}

A classic problem that appears in several scientific fields consists in determining a collective preference from the preferences of a group of individuals on a set of alternatives. The simplest situation happens when the individuals have to choose between two alternatives. However, in spite of being the simplest case, it is not trivial and the analysis of voting systems (henceforth called Social Welfare Functions, SWFs) between two alternatives has generated an abundant literature among academics. One

Bonifacio Llamazares

Departamento de Economía Aplicada, Instituto de Matemáticas (IMUVA), Universidad de Valladolid, Avda. Valle de Esgueva 6, 47011 Valladolid, Spain.

Tel.: +34 983186544

Fax: +34983423299

E-mail: boni@eco.uva.es 
of the most outstanding works on SWFs between two alternatives was realized by Fishburn (1973). One of his results, Fishburn (1973, p. 56), can be formulated as a characterization of the anonymous, neutral and monotonic SWFs. From this result, it is possible to characterize these SWFs by means of certain increasing functions.

Given that some of the SWFs most used in practice can be generated when the previous increasing functions are affine, the aim of this paper is to analyze the structure of the set formed by these functions. In this respect, in this paper we show that this set is convex and its extreme points are the functions that generate the following SWFs: simple majority, absolute majority, unanimous majority and Pareto majority. In addition to this, we show that the problem of selecting a SWF can be reduced to answering two simple questions: How many votes need an alternative for winning when the other alternative receives no vote? How many votes must an alternative gather for each vote obtained by the other alternative?

The paper is organized as follows. Section 2 provides the basic concepts and notation on SWFs. Moreover, the characterization result given by Fishburn is recalled. In Section 3 we give the main results of the paper. We conclude in Section 4.

\section{Preliminaries}

Let $N=\{1, \ldots, n\}$ be the set of voters, with $n \geq 3$, and $x, y$ two alternatives. Individual preferences between both alternatives are described by a profile $D=\left(d_{1}, \ldots, d_{n}\right)$, where $d_{i}$ is $1,-1$ or 0 depending on whether individual $i$ prefers $x$ to $y, y$ to $x$ or is indifferent between both alternatives. The set of profiles of preferences will be denoted by $\mathscr{D}$.

Given $D, D^{\prime} \in \mathscr{D}$ and $\sigma$ a permutation of $N$, we will use the following notation: $n^{+}(D)=\#\left\{i \in N \mid d_{i}=1\right\}, n^{-}(D)=\#\left\{i \in N \mid d_{i}=-1\right\},-D=\left(-d_{1}, \ldots,-d_{n}\right)$, $D_{\sigma}=\left(d_{\sigma(1)}, \ldots, d_{\sigma(n)}\right)$ and $D^{\prime} \geq D$ will mean $d_{i}^{\prime} \geq d_{i}$ for all $i \in N$.

For each profile of preferences, the collective preference will be obtained by means of SWFs.

Definition 1 A $S W F$ is a mapping $F: \mathscr{D} \longrightarrow\{-1,0,1\}$.

The possible values of $F, 1,0$, and -1 , have a similar interpretation as that in the case of individual preferences. Next we present some well-known properties of SWFs: Anonymity, neutrality and monotonicity. Anonymity, also referred to as equality and symmetry, means that collective preference depends only on the set of individual preferences, but not on which individuals have these preferences, i.e., all individuals are treated equally. Neutrality, also referred to as duality, says that if everyone reverses their preferences between $x$ and $y$, then the collective preference is also reversed, i.e., the alternatives are treated equally. Finally, monotonicity means that increased support for an alternative does not hurt this alternative.

Definition 2 Let $F$ be a SWF.

1. $F$ is anonymous if for all permutation $\sigma$ of $N$ and all profile $D \in \mathscr{D}$ we have $F\left(D_{\sigma}\right)=F(D)$.

2. $F$ is neutral if for all profile $D \in \mathscr{D}$ we have $F(-D)=-F(D)$. 
3. $F$ is monotonic if for all pair of profiles $D, D^{\prime} \in \mathscr{D}$ such that $D^{\prime} \geq D$ we have $F\left(D^{\prime}\right) \geq F(D)$.

It is worth noting that if $F$ is anonymous, then the value $F(D)$ depends only on $n^{+}(D)$ and $n^{-}(D)$. On the other hand, any neutral SWF is characterized by the set $F^{-1}(\{1\})$, since

$$
\begin{aligned}
F^{-1}(\{-1\}) & =\left\{D \in \mathscr{D} \mid-D \in F^{-1}(\{1\})\right\}, \\
F^{-1}(\{0\}) & =\mathscr{D} \backslash\left(F^{-1}(\{1\}) \cup F^{-1}(\{-1\})\right) .
\end{aligned}
$$

Anonymous, neutral and monotonic SWFs have been widely studied by Fishburn (1973). One of his results, Fishburn (1973, p. 56) can be given as a characterization of these functions. In what follows, given $a \in \mathbb{R},[a]$ will denote the integer part of $a$, i.e., the largest integer smaller than or equal to $a$.

Theorem 1 Let $F$ be a SWF. Then the following statements are equivalent:

1. $F$ is anonymous, neutral and monotonic.

2. There exist integers $r_{0} \leq r_{1} \leq \cdots \leq r_{[n / 2]}$ that satisfy $r_{i} \geq$ ifor all $i \in\{0, \ldots,[n / 2]\}$ and such that

$$
F(D)= \begin{cases}1 & \text { if } n^{+}(D)>r_{n^{-}(D)}, \\ -1 & \text { if } n^{-}(D)>r_{n^{+}(D)}, \\ 0 & \text { otherwise. }\end{cases}
$$

\section{The results}

Theorem 1 allows us to characterize the anonymous, neutral and monotonic SWFs by means of increasing functions that satisfy a certain property. For this, it is sufficient to take into account that the integers $r_{0}, r_{1}, \ldots, r_{[n / 2]}$ can be seen as the integer part of the values taken by a function. Moreover, given that any neutral SWF is characterized by the set $F^{-1}(\{1\})$, Fishburn's result can be given as follows.

Corollary 1 Let $F$ be a neutral SWF. Then the following statements are equivalent:

1. F is anonymous and monotonic.

2. There exists an increasing function $f:[0, n / 2] \longrightarrow[0, \infty)$ that satisfies $f(t) \geq t$ for all $t \in[0, n / 2]$ and such that

$$
F(D)=1 \Leftrightarrow n^{+}(D)>f\left(n^{-}(D)\right) .
$$

Proof

$(1) \Rightarrow(2)$ : By Theorem 1, there exist integers $r_{0} \leq r_{1} \leq \cdots \leq r_{[n / 2]}$ that satisfy $r_{i} \geq i$ for all $i \in\{0,1, \ldots,[n / 2]\}$. Therefore, the piecewise linear function $f$ such that $f(i)=r_{i}$ for all $i \in\{0,1, \ldots,[n / 2]\}$ and, if $n$ is odd, $f(n / 2)=\max \{n / 2, f([n / 2])\}$ satisfies the conditions of the theorem.

$(2) \Rightarrow(1)$ : The integers $r_{i}=[f(i)], i \in\{0,1, \ldots,[n / 2]\}$, satisfy $r_{0} \leq r_{1} \leq \cdots \leq$ $r_{[n / 2]}$ and $r_{i} \geq i$ for all $i \in\{0,1, \ldots,[n / 2]\}$. Therefore, by Theorem $1, F$ is anonymous and monotonic. 
Let $\mathscr{C}$ be the set of increasing functions $f:[0, n / 2] \longrightarrow[0, \infty)$ that satisfy $f(t) \geq t$ for all $t \in[0, n / 2]$. According to Corollary 1 , given $f \in \mathscr{C}$, we can associate with $f$ an anonymous, neutral and monotonic SWF $F_{f}$ defined by

$$
F_{f}(D)=1 \Leftrightarrow n^{+}(D)>f\left(n^{-}(D)\right) .
$$

It is worth noting that different functions $f, g \in \mathscr{C}$ can generate the same SWF. In order to characterize this kind of functions, we will use the following notation: Given $f \in \mathscr{C}, N_{f}=\{t \in\{0,1, \ldots,[n / 2]\} \mid t+[f(t)] \leq n-1\}$. The set $N_{f}$ is closely connected with the SWF $F_{f}$, as we show in the following lemma.

Lemma 1 Let $f \in \mathscr{C}$. Then the following statements are equivalent:

1. $t \in N_{f}$.

2. There exists $D \in \mathscr{D}$ such that $n^{-}(D)=t$ and $F_{f}(D)=1$.

Proof

(1) $\Rightarrow$ (2): Given $t \in N_{f}$, let $D \in \mathscr{D}$ a profile such that $n^{-}(D)=t$ and $n^{+}(D)=$ $[f(t)]+1$. This profile exists because $n^{-}(D)+n^{+}(D)=t+[f(t)]+1 \leq n$. Moreover, $F_{f}(D)=1$ since $n^{+}(D)>f\left(n^{-}(D)\right)$.

(2) $\Rightarrow(1)$ : Suppose that there exists $D \in \mathscr{D}$ such that $n^{-}(D)=t$ and $F_{f}(D)=1$. Since $F_{f}(D)=1$, we have $n^{+}(D)>f\left(n^{-}(D)\right)=f(t) \geq[f(t)]$. Therefore, $t+[f(t)]<$ $n^{-}(D)+n^{+}(D) \leq n$ and, consequently, $t \in N_{f}$.

Now, we are going to characterize the functions that generate the same SWFs.

Proposition 1 Let $f, g \in \mathscr{C}$. Then the following statements are equivalent:

1. $F_{f}=F_{g}$.

2. $N_{f}=N_{g}$ and $[f(t)]=[g(t)]$ for all $t \in N_{f}$.

Proof

(1) $\Rightarrow$ (2): First, we are going to prove that $N_{f}=N_{g}$. By Lemma $1, t \in N_{f}$ if and only if there exists $D \in \mathscr{D}$ such that $n^{-}(D)=t$ and $F_{f}(D)=1$. Since $F_{f}=F_{g}$, then there exists $D \in \mathscr{D}$ such that $n^{-}(D)=t$ and $F_{g}(D)=1$ and, again by Lemma 1, this is equivalent to $t \in N_{g}$.

Now, we are going to prove that $[f(t)]=[g(t)]$ for all $t \in N_{f}$. This is proven by contradiction. Suppose that there exists $t \in N_{f}$ such that $[f(t)] \neq[g(t)]$. We can suppose, without loss of generality, that $[f(t)]<[g(t)]$. Consider a profile $D \in \mathscr{D}$ such that $n^{-}(D)=t$ and $n^{+}(D)=[f(t)]+1$ (this profile exists because $n^{-}(D)+$ $\left.n^{+}(D)=t+[f(t)]+1 \leq n\right)$. For this profile we have $F_{f}(D)=1$ and $F_{g}(D)<1$; i.e., a contradiction.

(2) $\Rightarrow(1)$ : Since $F_{f}$ and $F_{g}$ are neutral, it is sufficient to prove that $F_{f}(D)=1$ if and only if $F_{g}(D)=1$ for all $D \in \mathscr{D}$. Let $D \in \mathscr{D}$ such that $F_{f}(D)=1$. By Lemma 1 , if $t=n^{-}(D)$, then $t \in N_{f}$, and, by definition of $F_{f}$ and $F_{g}$, we have:

$$
\begin{aligned}
F_{f}(D)=1 & \Rightarrow n^{+}(D)>f\left(n^{-}(D)\right) \Rightarrow n^{+}(D)>\left[f\left(n^{-}(D)\right)\right] \\
& \Rightarrow n^{+}(D)>\left[g\left(n^{-}(D)\right)\right] \Rightarrow n^{+}(D)>g\left(n^{-}(D)\right) \Rightarrow F_{g}(D)=1 .
\end{aligned}
$$

In a similar way we can prove that $F_{g}(D)=1 \Rightarrow F_{f}(D)=1$ for all $D \in \mathscr{D}$. 
The null SWF is defined by $F(D)=0$ for all $D \in \mathscr{D}$. It is easy to check that a function $f \in \mathscr{C}$ generates the null SWF if and only if $N_{f}=\emptyset$, which is equivalent to $f(0) \geq n$.

It is worth noting that the main SWFs used in practice can be generated by affine functions. For this reason, we are going to analyze the set formed by the affine functions that generate anonymous, neutral and monotonic SWFs. Firstly, given an affine function $f(t)=a t+b$, we are going to show the consequences that the conditions imposed on the function $f$ have on $a$ and $b$.

\section{Remark 1}

1. $f$ is increasing if and only if $a \geq 0$.

2. The condition $f(t) \geq t$ for all $t \in[0, n / 2]$ is equivalent to $b \geq 0$ and $b \geq(1-$ a) $(n / 2)$.

3. In order to avoid the null SWF, we consider $f(0)=b<n$.

4. We can only consider affine functions that satisfy the condition $a+b \leq n-1$ (If $a+b>n-1$, with $b<n$, then the function $g(t)=a^{\prime} t+b^{\prime}$, where $a^{\prime}=n-1-[b]$ and $b^{\prime}=[b]$, satisfies $N_{g}=N_{f}=\{0\}$ and $[g(0)]=[f(0)]$; that is, by Proposition 1 generates the same SWF as $f$ and $\left.a^{\prime}+b^{\prime} \leq n-1\right)$.

Consequently, we can restrict our study to the following set of affine functions:

$$
\mathscr{L}=\{a t+b \mid a \geq 0, b \geq 0,(1-a)(n / 2) \leq b \leq n-1-a\} .
$$

Notice that the SWFs generated through the affine functions of $\mathscr{L}$ are determined by the values of $a$ and $b$. The parameter $b$ shows the support that an alternative needs for winning $([b]+1$ votes) when the other alternative lacks support. Analogously, the parameter $a$ reflects the support that an alternative needs by each vote that the other alternative receives. Therefore, we only need to specify these parameters to select a SWF.

Next we are going to show some affine functions and the anonymous, neutral and monotonic SWFs that they generate.

\section{Remark 2}

1. When $f(t)=t$ we obtain simple majority:

$$
F(D)=1 \Leftrightarrow n^{+}(D)>n^{-}(D) .
$$

Simple majority has been widely studied and characterized by several authors. The first axiomatic characterization of this SWF was given by May (1952) and, after that, it has also been characterized, in the case of two alternatives, by Fishburn (1973, p. 58), Fishburn (1983), Aşan and Sanver (2002), Woeginger (2003, 2005), Miroiu (2004), Llamazares (2006), Xu and Zhong (2010) and Quesada (2013).

2. When $f(t)=n / 2$ we obtain absolute majority:

$$
F(D)=1 \Leftrightarrow n^{+}(D)>n / 2 \text {. }
$$

This majority has been characterized by Fishburn (1973, p. 60), Sanver (2006) and Quesada (2010). 
3. When $f(t)=n-1$ we obtain unanimous majority:

$$
F(D)=1 \Leftrightarrow n^{+}(D)=n \text {. }
$$

Unanimous majority has been characterized by Woeginger (2003), Llamazares (2006) and Quesada (2011).

4. When $f(t)=(n-1) t$ we obtain Pareto majority:

$$
F(D)=1 \Leftrightarrow n^{+}(D)>0 \text { and } n^{-}(D)=0 .
$$

Pareto majority has been characterized by Sen (1970, p. 76).

5. Given $\alpha \in[1 / 2,1)$, if $f(t)=\alpha n$, then we obtain the absolute special majority $A_{\alpha}$ :

$$
A_{\alpha}(D)=1 \Leftrightarrow n^{+}(D)>\alpha n \text {. }
$$

These rules are found between absolute majority, for $\alpha=1 / 2$, and unanimous majority, for $\alpha \geq(n-1) / n$. Absolute special majorities have been studied by Ferejohn and Grether (1974), and characterized by Fishburn (1973, p. 67) (without the neutrality assumption), Austen-Smith and Banks (1999, p. 80), Aşan and Sanver (2006), Houy (2007a) and Sanver (2009). On the other hand, according to (4) of Remark 1, if $\alpha \in((n-1) / n, 1)$, then $f(t)=\alpha n$ and $g(t)=((n-1) / n) n$ generate the same SWF; i.e., $A_{\alpha}=A_{\frac{n-1}{n}}$ for all $\alpha \in((n-1) / n, 1)$. Therefore, we can only consider $\alpha \in[1 / 2,(n-1) / n]^{n}$.

6 . Given $\beta \geq 1$, if $f(t)=\beta t$, then we obtain the relative special majority $R_{\beta}$ :

$$
R_{\beta}(D)=1 \Leftrightarrow n^{+}(D)>\beta n^{-}(D) .
$$

These rules are found between simple majority, for $\beta=1$, and Pareto majority, for $\beta \geq n-1$. Relative special majorities have been studied by Craven (1971), Fishburn (1973, p. 68) (without the neutrality assumption), Ferejohn and Grether (1974) and Jain $(1983,1986)$. On the other hand, according to (4) of Remark 1, if $\beta>n-1$, then $f(t)=\beta n$ and $g(t)=(n-1) t$ generate the same SWF; i.e., $R_{\beta}=R_{n-1}$ for all $\beta>n-1$. Therefore, we can only consider $\beta \in[1, n-1]$.

7. Given $\delta \in[0,1]$, if $f(t)=((1-\delta) /(1+\delta)) t+(\delta /(1+\delta)) n$, then we obtain the semi-strict majority $S_{\delta}$ :

$$
S_{\delta}(D)=1 \Leftrightarrow n^{+}(D)>\frac{1-\delta}{1+\delta} n^{-}(D)+\frac{\delta}{1+\delta} n .
$$

These rules are found between simple majority, for $\delta=0$, and absolute majority, for $\delta=1$. Semi-strict majorities have been studied by Pattanaik (1971, p. 54) (with an equivalent formulation and he called them $M$-rules).

8. Given $k \in\{0,1, \ldots, n-1\}$, if $f(t)=t+k$, then we obtain the majority based on difference of votes $M_{k}$ :

$$
M_{k}(D)=1 \Leftrightarrow n^{+}(D)>n^{-}(D)+k .
$$

These rules are found between simple majority, for $k=0$, and unanimous majority, $k=n-1$. Majorities based on difference of votes have been characterized by Llamazares (2006), Houy (2007b) and Sanver (2009). On the other hand, according to (4) of Remark $1, f(t)=t+n-1$ and $g(t)=n-1$ generate the same SWF, the unanimous majority. Therefore, we can only consider $k \in\{0,1, \ldots, n-2\}$. 
In the following theorem we show that the set $\mathscr{L}$ is convex and its extreme points are the functions that generate the following SWFs: simple majority, absolute majority, unanimous majority and Pareto majority.

Theorem 2 The set $\mathscr{L}$ is convex and its extreme points are the functions $f(t)=t$, $f(t)=n / 2, f(t)=n-1$ and $f(t)=(n-1) t$.

Proof Let $\mathscr{V}=\{a t+b \mid a, b \in \mathbb{R}\}$ and $\psi: \mathscr{V} \longrightarrow \mathbb{R}^{2}$ defined by $\psi(a t+b)=(a, b)$. Taking into account that $\mathscr{V}$ is a vector space and that the map $\psi$ is an isomorphism, the following results are derived from the theory of convex sets:

1. $\mathscr{L}$ is a convex set if and only if $\psi(\mathscr{L})$ is a convex set.

2. $a t+b$ is an extreme point of $\mathscr{L}$ if and only if $(a, b)$ is an extreme point of $\psi(\mathscr{L})$.

Therefore, in order to prove the theorem it is sufficient to show that $\psi(\mathscr{L})=$ $\{(a, b) \mid a \geq 0, b \geq 0,(1-a)(n / 2) \leq b \leq n-1-a\}$ is a convex set and that its extreme points are $(1,0),(0, n / 2),(0, n-1)$ and $(n-1,0)$. On the one hand, $\psi(\mathscr{L})$ is a convex set because it is a polyhedral set; on the other hand, it is easy to check (for instance, graphically; see Figure 1) that $(1,0),(0, n / 2),(0, n-1)$ and $(n-1,0)$ are extreme points of $\psi(\mathscr{L})$.

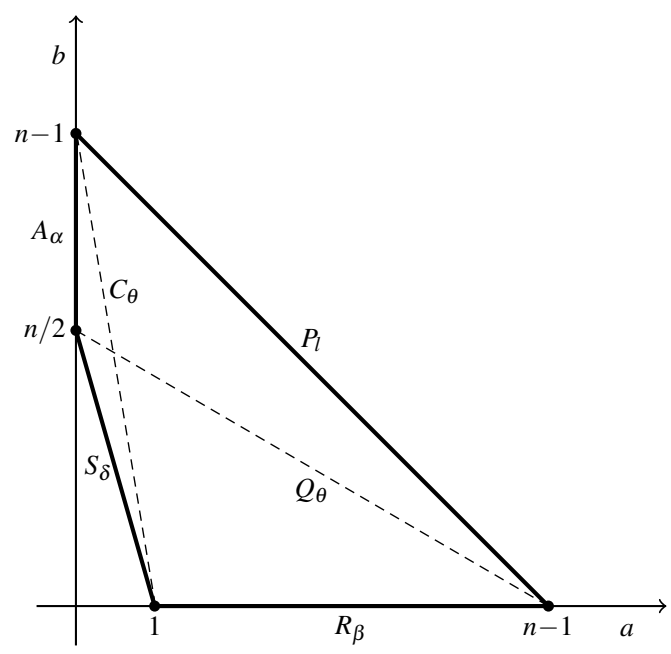

Fig. 1 The set $\psi(\mathscr{L})$ and different classes of majorities.

It is worth noting that some of the affine functions shown in Remark 2 are convex combinations of two extreme points of $\mathscr{L}$ (see Figure 1). For instance,

1. The functions that generate absolute special majorities are convex combinations of the functions that generate absolute and unanimous majorities:

$$
\alpha n=(1-\theta) \frac{n}{2}+\theta(n-1), \text { where } \theta=\frac{\alpha n-n / 2}{n / 2-1} .
$$


2. The functions that generate relative special majorities are convex combinations of the functions that generate simple and Pareto majorities:

$$
\beta t=(1-\theta) t+\theta(n-1) t, \text { where } \theta=\frac{\beta-1}{n-2} .
$$

3. The functions that generate semi-strict majorities are convex combinations of the functions that generate simple and absolute majorities:

$$
\frac{1-\delta}{1+\delta} t+\frac{\delta}{1+\delta} n=(1-\theta) t+\theta \frac{n}{2}, \text { where } \theta=\frac{2 \delta}{1+\delta} .
$$

It is possible to obtain three more families of SWFs generated by functions that are convex combinations of two extreme points of $\mathscr{L}$. Thus:

1. When we consider convex combinations of the functions that generate Pareto and unanimous majorities we obtain

$$
f(t)=(1-\theta)(n-1) t+\theta(n-1),
$$

with $\theta \in[0,1]$. If $l=[\theta(n-1)]$, then $l \in\{0,1, \ldots, n-1\}$ and the majority generated by $f$ is

$$
P_{l}(D)=1 \Leftrightarrow n^{+}(D)>l \text { and } n^{-}(D)=0 .
$$

2. When we consider convex combinations of the functions that generate Pareto and absolute majorities we obtain

$$
f(t)=(1-\theta)(n-1) t+\theta \frac{n}{2}
$$

with $\theta \in[0,1]$. In this case, the majority generated by $f$ is

$$
Q_{\theta}(D)=1 \Leftrightarrow n^{+}(D)>(1-\theta)(n-1) n^{-}(D)+\theta \frac{n}{2} .
$$

3. When we consider convex combinations of the functions that generate simple and unanimous majorities we obtain

$$
f(t)=(1-\theta) t+\theta(n-1),
$$

with $\theta \in[0,1]$. In this case, the majority generated by $f$ is

$$
C_{\theta}(D)=1 \Leftrightarrow n^{+}(D)>(1-\theta) n^{-}(D)+\theta(n-1) .
$$

It is worth noting that the functions that generate majorities based on difference of votes, $f(t)=t+k$ (with $k \in\{0,1, \ldots, n-2\}$ ), are convex combinations of the functions that generate simple, Pareto and unanimous majorities:

$$
t+k=\left(1-\frac{k}{n-2}\right) t+\frac{k}{(n-2)(n-1)}(n-1) t+\frac{k}{n-1}(n-1) .
$$




\section{Concluding remarks}

Social choice problems are very common in real life and appear in many different fields. One of the most important issues in this subject is how to choose the decision rule to be used. One possibility is to choose the decision rule according to its properties. For this, the axiomatic characterizations given in the literature are essential. When only two alternatives are in conflict, Corollary 1 and the set of affine functions $\mathscr{L}$ (to the best of our knowledge, affine functions are the only used in real life) provide another easy possibility: It is sufficient to determine the parameters $a$ and $b$ (we recall that $b$ shows the support that an alternative needs for winning, $[b]+1$ votes, when the other alternative lacks support, while $a$ reflects the support that an alternative needs by each vote that the other alternative receives). Lastly, knowing that certain majorities are convex combination of simple, absolute, unanimous or Pareto majorities could also be useful. For instance, consider the case of semi-strict majorities, $S_{\delta}$. As we have seen, they are convex combination of simple and absolute majorities, $f(t)=(1-\theta) t+\theta \frac{n}{2}$. Since $\theta=\frac{2 \delta}{1+\delta}$, when $\delta$ is a value close to 0 , so is $\theta$, and the behavior of $S_{\delta}$ is similar to simple majority. Instead, when $\delta$ is a value close to 1 , so is $\theta$, and the behavior of $S_{\delta}$ is similar to absolute majority.

Acknowledgements This work is partially supported by the Spanish Ministry of Economy and Competitiveness (Project ECO2012-32178) and ERDF. The author is grateful to an anonymous referee for valuable comments and suggestions.

\section{References}

Aşan G, Sanver MR (2002) Another characterization of the majority rule. Econ Lett 75:409-413

Aşan G, Sanver MR (2006) Maskin monotonic aggregation rules. Econ Lett 91:179183

Austen-Smith D, Banks JS (1999) Positive Political Theory I: Collective Preference. The University of Michigan Press, Ann Arbor

Craven J (1971) Majority voting and social choice. Rev Econ Stud 38:265-267

Ferejohn JA, Grether DM (1974) On a class of rational social decisions procedures. J Econ Theory 8:471-482

Fishburn PC (1973) The Theory of Social Choice. Princeton University Press, Princeton

Fishburn PC (1983) A new characterization of simple majority. Econ Lett 13:31-35

Houy N (2007a) A new characterization of absolute qualified majority voting. Econ Bull 4:1-8

Houy N (2007b) Some further characterizations for the forgotten voting rules. Math Soc Sci 53:111-121

Jain SK (1983) Necessary and sufficient conditions for quasi-transitivity and transitivity of special majority rules. Keio Econ Stud 20:55-63 
Jain SK (1986) Special majority rules: Necessary and sufficient condition for quasitransitivity with quasi-transitive individual preferences. Soc Choice Welfare 3:99106

Llamazares B (2006) The forgotten decision rules: Majority rules based on difference of votes. Math Soc Sci 51:311-326

May KO (1952) A set of independent necessary and sufficient conditions for simple majority decisions. Econometrica 20:680-684

Miroiu A (2004) Characterizing majority rule: from profiles to societies. Econ Lett 85:359-363

Pattanaik PK (1971) Voting and Collective Choice. Cambridge University Press, Cambridge

Quesada A (2010) Monotonicity + efficiency + continuity = majority. Math Soc Sci 60:149-153

Quesada A (2011) Parallel axiomatizations of majority and unanimity. Econ Lett 111:151-154

Quesada A (2013) The majority rule with a chairman. Soc Choice Welfare 40:679691

Sanver MR (2006) Nash implementation of the majority rule. Econ Lett 91:369-372

Sanver MR (2009) Characterizations of majoritarianism: a unified approach. Soc Choice Welfare 33:159-171

Sen AK (1970) Collective Choice and Social Welfare. Holden-Day, San Francisco Woeginger GJ (2003) A new characterization of the majority rule. Econ Lett 81:8994

Woeginger GJ (2005) More on the majority rule: Profiles, societies, and responsiveness. Econ Lett 88:7-11

$\mathrm{Xu}$ Y, Zhong Z (2010) Single profile of preferences with variable societies: A characterization of simple majority rule. Econ Lett 107:119-121 